\title{
Human Papillomavirus Distribution among Women in Western Shandong Province, East China using Reverse Blot Hybridization Assay
}

\author{
Dongsup Lee, ${ }^{1, \S}$, Geehyuk Kim ${ }^{2, \S}$, Sunghyun Kim ${ }^{3}$, Sunyoung Park ${ }^{2}$, Hye-young Wang ${ }^{4}$, \\ Sangjung Park ${ }^{5}$, Lin Han ${ }^{6}$, Ren Yubo ${ }^{6}$, Yingxue Li ${ }^{6}$, Kwang Hwa Park ${ }^{7, \dagger}$ and Hyeyoung Lee ${ }^{2, \dagger}$ \\ ${ }^{I}$ Department of Clinical Laboratory Science, Hyejeon College, Hongseong, Chungnam 350-702, Korea; \\ ${ }^{2}$ Department of Biomedical Laboratory Science, College of Health Sciences, Yonsei University, Wonju, \\ Gangwon 220-710, Korea; ${ }^{3}$ Department of Clinical Laboratory Science, College of Health Sciences, \\ Catholic University of Pusan, Busan 609-757, Korea; ${ }^{4}$ Optipham M\&D, Inc., Wonju Eco Environmental \\ Technology Center, Wonju, Gangwon 220-710, Korea, ${ }^{5}$ Department of Clinical Laboratory Science, \\ College of Medical Science, Daegu Haany University, Daegu 712-715, Korea; ${ }^{6}$ Department of Pathology, \\ Liaocheng School of Clinical Medicine, Taishan Medical University, Liaocheng, Shandong 11206, China; \\ ${ }^{7}$ Department of Pathology, Yonsei University Wonju College of Medicine, Wonju, Gangwon 220-701, Korea
}

Cervical cancer is the third most common cancer in women worldwide and there is a significant association between human papillomavirus (HPV) infection and cervical cancer. Certain HPV groups, labeled high-risk (HR) HPV groups, are strongly associated with malignancies of the human cervix. HPV prevalence and genotype distribution were analyzed using the REBA HPV-ID ${ }^{\circledR}$ (YD Diagnostics, Yongin, Korea) assay based on the reverse blot hybridization assay (REBA) with a total of 324 liquid-based cytology samples from women in Western Shandong Province, East China and results were compared with cytological diagnosis. Most of the HPV genotypes that were detected in high-grade cervical lesions were HR-HPV genotypes such as HPV 16, 18, 33, 53, and 58. The prevalence of these HR-HPV genotypes increased in high-grade cervical lesions. However, from low- to high-grade cervical lesions, the ability to detect LR-HPV genotypes decreased. Additionally, in general, the single HPV genotype infection rate increases in proportion to the severity of the lesion. The study findings suggest that a currently available preventive vaccine against HPV 16 and 18 may have limited effectiveness for prevention of all HPV infection in this province. Finally, based on these findings, these data could guide national or regional vaccination programs in the Western Shandong Province of East China to substantially reduce the burden of cervical lesions.

Key Words: Human papillomavirus, Prevalence, Genotype-distribution, REBA HPV-ID ${ }^{\circledR}$, Western Shandong Province

\footnotetext{
*Received: May 19, 2015 / Revised: June 5, 2015 Accepted: June 22, 2015

$\S_{\text {Equal contributors. }}$

${ }^{\dagger}$ Corresponding author: Hyeyoung Lee. Department of Biomedical Laboratory Science, College of Health Sciences, Yonsei University, 1 yonseidae-gil, Wonju, Gangwon 220-710, Korea.

Tel: +82-33-760-2740, Fax: +82-33-760-2561

e-mail: hyelee@yonsei.ac.kr

†Corresponding author: Kwang Hwa Park. Department of Pathology, Wonju College of Medicine, Yonsei University, 20 Ilsan-ro, Wonjui, Gangwon 220-701, Korea.

Tel: +82-33-741-1556, Fax: +82-33-731-6590

e-mail: abba@yonsei.ac.kr

(C)The Korean Society for Biomedical Laboratory Sciences. All rights reserved.
}

\section{INTRODUCTION}

Cervical cancer is the third most common cancer in women worldwide (Ferlay et al., 2010). There are 75,500 new cases and 34,000 deaths annually in developing countries such as China (Zhao et al., 2012) (Dunne et al., 2007). Recent studies estimated that there is a significant association between human papillomavirus (HPV) infection and cervical cancer and precancerous lesions of the cervix (Bosch et al., 
2003). More than 100 HPV genotypes have been identified, and certain HPV groups, such as high-risk (HR) HPV groups, have been strongly associated with malignancies of the human cervix. HPV 16, 18, 31, 33, 35, 39, 45, 51, 52, 56, 58,59 and 68 are well-known carcinogenic HR-HPV genotypes and HPV 26, 30, 34, 53, 66, 67, 69, 70, 73, 82, 85 and 97 are also considered to be carcinogenic HPV genotypes (Agarossi et al., 2009) (Bouvard et al., 2009).

However, HPV prevalence and genotype distribution in invasive cervical and precancerous lesions vary, depending on the country and province. For instance, previous studies reported that HPV 16, 18, 33, 45, and 31 were the most prevalent HPV genotypes in the world; HPV 16, 18, 33, 31, 45 in Europe; HPV 16, 18, 31, 33, and 45 were the most common HPV genotypes in North America; HPV 16, 18, 33, 45, and 35 were the most prevalent HPV genotypes in Africa. However, in Asia, the most prevalent HPV genotypes were HPV 16, 18, 58, 33, and 52. The HPV genotypedistribution between Asia and other continents differs (Bosch et al., 2008). In addition, many previous studies have investigated the HPV prevalence and genotype distribution in Asian countries. In the Korea, the most common HPV genotypes were HPV 16, 18, 33, 53, 56, and 58 (Kim et al., 2012a). Meanwhile, HPV 16, 18, 33, 52, and 58 are the most prevalent HPV genotypes in Beijing (Ding et al., 2014). Many studies show a difference even within Asia.

This is the first study reporting the distribution of HPV genotypes in Shandong, China. In this study, the REBA HPV-ID ${ }^{\circledR}$ assay (YD diagnostics, Yongin, Korea), which is a reverse blot hybridization-based assay (REBA) was employed to reveal the distribution of 15 HR-HPV genotypes (HPV 16, 18, 31, 33, 35, 39, 45, 51, 52, 53, 56, 58, 59, 66, and 68) and 10 low-risk (LR) HPV genotypes (HPV 6, $11,42,43,44,70,72,81,84$, and 87). This approach was used to detect HPV genotypes in uterine cervical liquidbased cytology samples from a total of 324 Eastern Chinese Women.

\section{MATERIALS AND METHODS}

\section{Patients and clinical samples}

A total of 324 liquid-based cytology samples were collected at Liaocheng People's Hospital in China between January 2013 and December 2013 (approval no. 2011-0038). Liaocheng People's Hospital is located in the western region of Shandong Province and is a comprehensive tertiary health care hospital. It has 3500 inpatient beds and 20 clinical cytopathologists. By drawing a random sample from a larger population, the sample is representative of the larger group and less likely to be subject to bias.

\section{Cytological diagnosis}

Liquid-based cytology slides (Pap smears) were evaluated according to the 2001 Bethesda System designed by cytopathologists and pathologists. Cytological cases of benign (within normal limit, reactive change due to inflammation, fungal infection, and atrophy), atypical squamous cells of undetermined significance (ASC-US), atypical squamous cells - cannot exclude HSIL (ASC-H), low-grade squamous intraepithelial lesion (LSIL), HSIL, and SCC were included. The remaining fluid samples were stored at $4{ }^{\circ} \mathrm{C}$ after cytology slide preparation and before DNA extraction.

\section{DNA extraction}

DNA extraction was performed using the HPV DNA Extraction Solution (M\&D, Wonju, Korea), according to the manufacturer's recommendations. Briefly, remaining clinical samples were collected in 50-mL tubes and vortexed for about $1 \mathrm{~min}$. The volume was adjusted to $40 \mathrm{ml}$ with PBS (pH 7.2) and centrifuged at $2,000 \mathrm{~g}$ at $4{ }^{\circ} \mathrm{C}$ for $30 \mathrm{~min}$. The supernatant was discarded and $300 \sim 500 \mu \mathrm{L}$ of sterile distilled water (DW) was added to the pellet, vortexed, transferred to a $1.5-\mathrm{mL}$ Eppendorf tube, and then centrifuged at $17,590 \mathrm{~g}, 4^{\circ} \mathrm{C}$ for $5 \mathrm{~min}$. The supernatant was discarded. DNA extraction solution $(100 \mu \mathrm{L})$ was added to the pellet, vortexed for $1 \mathrm{~min}$, and incubated at $56^{\circ} \mathrm{C}$ for 15 min with intermittent tube tapping. After incubation, samples were boiled for $10 \mathrm{~min}$ on a boiling water bath and centrifuged at $17,590 \mathrm{~g}$ for $3 \mathrm{~min}\left(25^{\circ} \mathrm{C}\right)$, and then the supernatant ( 3 to $5 \mu \mathrm{L}$ ) was used as a template for PCR.

\section{HPV genotyping using PCR-reverse blot hybridization assay (PCR-REBA)}

REBA HPV-ID ${ }^{\circledR}$ test (YD Diagnostic, Yongin, Korea) 
Table 1. Cytological diagnosis in different age groups

\begin{tabular}{lccccccc}
\hline \hline $\begin{array}{c}\text { Cytological } \\
\text { diagnosis }\end{array}$ & $\begin{array}{c}\text { Normal } \\
(\mathrm{n}=79)\end{array}$ & $\begin{array}{c}\text { ASC-US } \\
(\mathrm{n}=96)\end{array}$ & $\begin{array}{c}\text { LSIL } \\
(\mathrm{n}=59)\end{array}$ & $\begin{array}{c}\text { ASC-H } \\
(\mathrm{n}=19)\end{array}$ & $\begin{array}{c}\text { HSIL } \\
(\mathrm{n}=67)\end{array}$ & $\begin{array}{c}\text { SCC } \\
(\mathrm{n}=4)\end{array}$ & $\begin{array}{c}\text { Total } \\
(\mathrm{n}=324)\end{array}$ \\
\hline Median age & 42 & 45.5 & 36 & 44 & 44 & 56.5 & 43 \\
\hline Age (years) & Number $(\%)$ & Number $(\%)$ & Number $(\%)$ & Number $(\%)$ & Number $(\%)$ & Number $(\%)$ & Number $(\%)$ \\
$20 \sim 29$ & $15(19.0)$ & $10(10.4)$ & $16(27.1)$ & $1(5.3)$ & $8(11.9)$ & $0(0)$ & $50(15.4)$ \\
$30 \sim 39$ & $17(21.5)$ & $15(15.6)$ & $16(27.1)$ & $3(15.8)$ & $13(19.4)$ & $0(0)$ & $64(19.8)$ \\
$40 \sim 49$ & $23(29.1)$ & $28(29.2)$ & $14(23.7)$ & $8(42.1)$ & $24(35.8)$ & $0(0)$ & $97(29.9)$ \\
$50 \sim 59$ & $17(21.5)$ & $27(28.1)$ & $8(13.6)$ & $6(31.6)$ & $16(23.9)$ & $3(75.0)$ & $77(23.8)$ \\
$60 \sim 69$ & $5(6.3)$ & $12(12.5)$ & $4(6.8)$ & $1(5.3)$ & $5(7.5)$ & $1(25.0)$ & $28(8.6)$ \\
$70 \sim 79$ & $2(2.5)$ & $4(4.2)$ & $1(1.7)$ & $0(0)$ & $1(1.5)$ & $0(0)$ & $8(2.5)$ \\
$80 \sim 89$ & $0(0)$ & $0(0)$ & $0(0)$ & $0(0)$ & $0(0)$ & $0(0)$ & $0(0)$ \\
\hline
\end{tabular}

ASC-US, atypical squamous cells of undetermined significance; LSIL, low grade squamous intraepithelial lesion; ASC-H, atypical squamous cells - cannot exclude HSIL; HSIL, high grade squamous intraepithelial lesion; SCC, squamous cell carcinoma

based on PCR-REBA was performed for HPV genotyping. ${ }^{14}$ This genotyping method required nested PCR to amplify the target regions including MY11 and MY9, and GP5 and GP6 with two pairs of primer. After PCR amplification, hybridization and washing processes were performed according to the manufacturer's instructions. In brief, the PCR products with biotin were denatured at $25^{\circ} \mathrm{C}$ for $5 \mathrm{~min}$ in denaturation solution and diluted in $970 \mu \mathrm{L}$ of hybridization solution on the REBA membrane strip in the provided blotting tray. Denatured single-stranded PCR products were hybridized to the probes on a strip at $50^{\circ} \mathrm{C}$ for $30 \mathrm{~min}$. The strips were then washed twice with gentle shaking in $1 \mathrm{~mL}$ of washing solution for $10 \mathrm{~min}$ at $50^{\circ} \mathrm{C}$, incubated at $25^{\circ} \mathrm{C}$ with $1: 2,000$ diluted streptavidin-alkaline phosphatase (AP) conjugate (Roche Diagnostics, Mannheim, Germany) in conjugate diluents solution (CDS) for $30 \mathrm{~min}$, and finally washed twice with $1 \mathrm{~mL} C D S$ at room temperature for $1 \mathrm{~min}$. The colorimetric hybridization signals were visualized by addition of a 1:50 dilution of AP-mediated staining solution, NBT/ BCIP (Roche Diagnostics) and incubation until color change was detected. Finally, the band pattern was read and interpreted.

\section{RESULTS}

\section{Cytological diagnosis}

Three hundred and twenty-four sample specimens were classified into six categories by cytological criteria: 4 (1.2\%) SCC, 67 (20.7\%) HSIL, 19 (5.9\%) ASC-H, 59 (18.2\%) LSIL, 96 (29.6\%) ASC-US, and 79 (24.4\%) normal (Table 1). Overall, patients in their $40 \mathrm{~s}$ had the highest percentage of HPV.

\section{HPV prevalence and high risk HPV prevalence according to cytological grade}

The overall HPV prevalence among 324 cytological grade samples was 259 cases (79.9\%). The HPV prevalence was 4 cases $(100 \%)$ for 4 SCC samples, 62 cases (92.5\%) for 67 HSIL, 18 cases $(94.7 \%)$ for 19 ASC-H, 55 cases $(93.2 \%)$ for 59 LSIL, 79 cases (82.3\%) for 96 ASC-US, and 41 cases (51.9\%) for 79 normal. High risk (HR) HPV prevalence among all cytological grade samples was 162 cases $(50.5 \%)$ and 4 cases $(100 \%)$ for 4 SCC samples, 58 cases $(86.6 \%)$ for 67 HSIL, 17 cases (89.5\%) for 19 ASC-H, 39 cases (66.1\%) for 59 LSIL, 33 cases $(34.4 \%)$ for 96 ASC-US, and 11 cases (13.9\%) for 79 normal (Table 2).

\section{HPV single infection and multiple infection patterns using REBA assay}

Among a total of 324 cervical liquid cytological samples, 135 cases $(41.7 \%)$ were infected with a single HPV genotype, 33 cases $(10.2 \%)$ were infected with multiple HPV genotypes (infected with more than two HPV genotypes), 91 cases $(28.1 \%)$ were infected with other HPV genotypes, 
Table 2. Positive HPV rates by cytological results

\begin{tabular}{cccccccc}
\hline \hline & $\begin{array}{c}\text { Normal } \\
(\mathrm{n}=79)\end{array}$ & $\begin{array}{c}\text { ASC-US } \\
(\mathrm{n}=96)\end{array}$ & $\begin{array}{c}\text { LSIL } \\
(\mathrm{n}=59)\end{array}$ & $\begin{array}{c}\text { ASC-H } \\
(\mathrm{n}=19)\end{array}$ & $\begin{array}{c}\text { HSIL } \\
(\mathrm{n}=67)\end{array}$ & $\begin{array}{c}\text { SCC } \\
(\mathrm{n}=4)\end{array}$ & $\begin{array}{c}\text { Total } \\
(\mathrm{n}=324)\end{array}$ \\
Cytological diagnosis & $\begin{array}{c}\text { Number } \\
(\%)\end{array}$ & $\begin{array}{c}\text { Number } \\
(\%)\end{array}$ & $\begin{array}{c}\text { Number } \\
(\%)\end{array}$ & $\begin{array}{c}\text { Number } \\
(\%)\end{array}$ & $\begin{array}{c}\text { Number } \\
(\%)\end{array}$ & $\begin{array}{c}\text { Number } \\
(\%)\end{array}$ & $\begin{array}{c}\text { Number } \\
(\%)\end{array}$ \\
\hline HPV positive & $41(51.9)$ & $79(82.3)$ & $55(93.2)$ & $18(94.7)$ & $62(92.5)$ & $4(100.0)$ & $259(79.9)$ \\
HR-HPV & $11(13.9)$ & $33(34.4)$ & $39(66.1)$ & $17(89.5)$ & $58(86.6)$ & $4(100.0)$ & $162(50.5)$ \\
LR-HPV & $1(1.3)$ & $2(2.1)$ & $2(3.4)$ & $1(5.3)$ & $0(0)$ & $0(0)$ & $6(1.9)$ \\
Other HPV & $29(36.7)$ & $44(45.8)$ & $14(23.7)$ & $0(0)$ & $4(6.0)$ & $0(0)$ & $91(28.1)$ \\
HPV negative & $38(48.1)$ & $17(17.7)$ & $4(6.8)$ & $1(5.3)$ & $5(7.5)$ & $0(0.0)$ & $65(20.1)$ \\
\hline
\end{tabular}

ASC-US, atypical squamous cells of undetermined significance; LSIL, low grade squamous intraepithelial lesion; ASC-H, atypical squamous cells - cannot exclude HSIL; HSIL, high grade squamous intraepithelial lesion; SCC, squamous cell carcinoma

Table 3. Multiplicity of HPV infection according to cytological diagnosis

\begin{tabular}{lccccccc}
\hline \hline & $\begin{array}{c}\text { Normal } \\
(\mathrm{n}=79)\end{array}$ & $\begin{array}{c}\text { ASC-US } \\
(\mathrm{n}=96)\end{array}$ & $\begin{array}{c}\text { LSIL } \\
(\mathrm{n}=59)\end{array}$ & $\begin{array}{c}\text { ASH-H } \\
(\mathrm{n}=19)\end{array}$ & $\begin{array}{c}\text { HSIL } \\
(\mathrm{n}=67)\end{array}$ & $\begin{array}{c}\text { SCC } \\
(\mathrm{n}=4)\end{array}$ & $\begin{array}{c}\text { Total } \\
(\mathrm{n}=324)\end{array}$ \\
& $\begin{array}{c}\text { Number } \\
(\%)\end{array}$ & $\begin{array}{c}\text { Number } \\
(\%)\end{array}$ & $\begin{array}{c}\text { Number } \\
(\%)\end{array}$ & $\begin{array}{c}\text { Number } \\
(\%)\end{array}$ & $\begin{array}{c}\text { Number } \\
(\%)\end{array}$ & $\begin{array}{c}\text { Number } \\
(\%)\end{array}$ & $\begin{array}{c}\text { Number } \\
(\%)\end{array}$ \\
\hline Single infection & $10(12.7)$ & $27(28.1)$ & $29(49.2)$ & $14(73.7)$ & $52(77.6)$ & $3(75.0)$ & $135(41.7)$ \\
HR- HPV & $9(11.4)$ & $24(25.0)$ & $27(45.8)$ & $13(68.4)$ & $52(77.6)$ & $3(75.0)$ & $128(39.5)$ \\
LR-HPV & $1(1.3)$ & $3(3.1)$ & $2(3.4)$ & $1(5.3)$ & $0(0)$ & $0(0)$ & $7(2.2)$ \\
Multiple infection & $2(2.5)$ & $8(8.3)$ & $12(20.3)$ & $4(21.1)$ & $6(9.0)$ & $1(25.0)$ & $33(10.2)$ \\
HR-HPV & $0(0)$ & $5(5.2)$ & $7(11.9)$ & $2(10.5)$ & $6(9.0)$ & $0(0)$ & $20(6.2)$ \\
LR-HPV & $0(0)$ & $0(0)$ & $0(0)$ & $0(0)$ & $0(0)$ & $0(0)$ & $0(0)$ \\
HR- \& LR-HPV mixed infection & $2(2.5)$ & $3(3.1)$ & $5(8.5)$ & $2(10.5)$ & $0(0)$ & $1(25.0)$ & $13(4.0)$ \\
Other HPV genotypes & $29(36.7)$ & $44(45.8)$ & $14(23.7)$ & $0(0)$ & $4(6.0)$ & $0(0)$ & $91(28.1)$ \\
Negative & $38(48.1)$ & $17(17.7)$ & $4(6.8)$ & $1(5.3)$ & $5(7.5)$ & $0(0)$ & $65(20.1)$ \\
\hline
\end{tabular}

ASC-US, atypical squamous cells of undetermined significance; LSIL, low grade squamous intraepithelial lesion; ASC-H, atypical squamous cells cannot exclude HSIL; HSIL, high grade squamous intraepithelial lesion; SCC, squamous cell carcinoma

and 65 cases $(20.1 \%)$ were HPV negative (Table 3$)$.

Among 135 single HPV genotype infection cases, 128 cases $(39.5 \%)$ were infected with HR-HPV genotypes and 7 cases $(2.2 \%)$ were infected with LR-HPV genotypes. Among 33 multiple HPV genotype infection cases, 20 cases (6.2\%) were infected with HR-HPV genotypes and 13 cases (4.0\%) were infected with both HR- and LR-HPV genotypes (HR- and LR-HPV genotype mixed infection) (Table 3).

Among 4 SCC cytological samples, 3 cases (75.0\%) were infected with a single HR-HPV genotype and 1 case involved a HR- and LR-HPV genotype mixed infection. Among 67 HSIL cytological samples, 52 cases (77.6\%) were single HR-HPV genotype infections, 6 cases $(9.0 \%)$ were multiple HR-HPV genotype infections, 4 cases (6.0\%) were other HPV genotype infections, and 5 cases (7.5\%) were HPV negatives. Among 19 ASC-H cytological samples, 13 cases $(68.4 \%)$ were single HR-HPV genotype infections, 2 cases $(10.5 \%)$ were multiple HR-HPV genotype infections, 2 cases $(10.5 \%)$ were HR- and LR-HPV genotype mixed infections, 1 case $(5.3 \%)$ was single LR-HPV, and 1 case (5.3\%) was HPV negative. Among 59 LSIL cytological samples, 27 cases $(45.8 \%)$ were single HR-HPV genotype infections, 7 cases (11.9\%) were multiple HR-HPV genotype infections, 5 cases $(8.5 \%)$ were HR- and LR-HPV 
Table 4. HPV genotype distribution according to cytological results

\begin{tabular}{|c|c|c|c|c|c|c|c|c|}
\hline & $\begin{array}{c}\text { HPV } \\
\text { Subtype }\end{array}$ & $\begin{array}{c}\text { Normal } \\
(\%)\end{array}$ & $\begin{array}{c}\text { ASC-US } \\
(\%)\end{array}$ & $\begin{array}{c}\text { LSIL } \\
(\%)\end{array}$ & $\begin{array}{c}\text { ASC-H } \\
(\%)\end{array}$ & $\begin{array}{c}\text { H-SIL } \\
(\%)\end{array}$ & $\begin{array}{l}\text { SCC } \\
(\%)\end{array}$ & $\begin{array}{c}\text { Total } \\
(\%)\end{array}$ \\
\hline \multirow{15}{*}{ High risk } & 16 & $3(21.4)$ & $11(24.4)$ & $9(17.0)$ & $9(4.5)$ & $41(64.1)$ & $2(40.0)$ & 75 (37.3) \\
\hline & 18 & $5(35.7)$ & $5(11.1)$ & $5(9.4)$ & $1(5.0)$ & 1 (1.6) & $1(20.0)$ & $18(9.0)$ \\
\hline & 31 & & $1(2.2)$ & & & & & $1(0.5)$ \\
\hline & 33 & & $2(4.4)$ & $1(1.9)$ & $1(5.0)$ & $6(9.4)$ & & $10(5.0)$ \\
\hline & 35 & $1(7.1)$ & $2(4.4)$ & $1(1.9)$ & $3(15.0)$ & $1(1.6)$ & & $8(4.0)$ \\
\hline & 39 & & $2(4.4)$ & $2(3.8)$ & & & & $4(2.0)$ \\
\hline & 45 & & & $2(3.8)$ & & $1(1.6)$ & & $3(1.5)$ \\
\hline & 51 & & & $2(3.8)$ & & & & $2(1.0)$ \\
\hline & 52 & $1(7.1)$ & $1(2.2)$ & $2(3.8)$ & & $2(3.1)$ & & $6(3.0)$ \\
\hline & 53 & & $7(15.6)$ & $8(15.1)$ & $1(5.0)$ & $3(4.7)$ & $1(20.0)$ & $20(10.0)$ \\
\hline & 56 & & $1(2.2)$ & $2(3.8)$ & & $3(4.7)$ & & $6(3.0)$ \\
\hline & 58 & & $4(8.9)$ & $4(7.5)$ & $2(10.0)$ & $5(7.8)$ & & $15(7.5)$ \\
\hline & 66 & $1(7.1)$ & $3(6.7)$ & $5(9.4)$ & & $1(1.6)$ & & $10(5.0)$ \\
\hline & 69 & & & $1(1.9)$ & & & & $1(0.5)$ \\
\hline & 73 & & & $2(3.8)$ & & & & $2(1.0)$ \\
\hline \multirow{6}{*}{ Low risk } & 6 & & & $1(1.9)$ & $1(5.0)$ & & & $2(1.0)$ \\
\hline & 11 & $2(14.3)$ & $1(2.2)$ & $1(1.9)$ & $1(5.0)$ & & & $5(2.5)$ \\
\hline & 40 & & $1(2.2)$ & $1(1.9)$ & & & & $2(1.0)$ \\
\hline & 43 & & $1(2.2)$ & & & & & $1(0.5)$ \\
\hline & 54 & $1(7.1)$ & $3(6.7)$ & $3(5.7)$ & $1(5.0)$ & & $1(20.0)$ & $9(4.5)$ \\
\hline & 84 & & & $1(1.9)$ & & & & $1(0.5)$ \\
\hline Total & 14 & 45 & 53 & 20 & 64 & 5 & 201 & \\
\hline
\end{tabular}

ASC-US, atypical squamous cells of undetermined significance; LSIL, low grade squamous intraepithelial lesion; ASC-H, atypical squamous cells - cannot exclude HSIL; HSIL, high grade squamous intraepithelial lesion; SCC, squamous cell carcinoma

genotype mixed infections, 2 cases (3.4\%) were single LRHPV, 14 cases (23.7\%) were other HPV genotype infections, and 4 cases (6.8\%) were HPV negative. Among 59 ASCUS cytological samples, 24 cases $(25.0 \%)$ were single HRHPV genotype infections, 5 cases $(5.2 \%)$ were multiple HR-HPV genotype infections, 3 cases (3.1\%) were HR- and LR-HPV genotype mixed infections, 3 cases (3.1\%) were single LR-HPV, 44 cases (45.8\%) were other HPV genotype infections, and 17 cases (17.7\%) were HPV negative. Among 79 normal cytological samples, 9 cases (11.4\%) were single HR-HPV genotype infections, 2 cases (2.5\%) were HR- and LR-HPV genotype mixed infections, 1 case (1.3\%) was a single LR-HPV, 29 cases $(36.7 \%)$ were other HPV genotype infections, and 38 cases (48.1\%) were HPV negative (Table 3).

\section{HPV genotype distribution according to cytological results}

The distribution of these HPV genotypes varied with cytological diagnosis is shown (Table 4). Among the total of 201 infections, HPV 16 (37.3\%), HPV 53 (10.0\%), HPV 18 (9.0\%), HPV 58 (7.5\%), HPV 33 and 66 (5.0\%), HPV 54 (4.5\%), HPV 35 (4.0\%) HPV 11 (2.5\%), HPV 39 (2.0\%) and HPV 45 (1.5\%) and HPV 31, HPV 51, HPV 69, HPV 73, HPV 6, HPV 40, HPV 43, HPV 84 (below 1.0\%) were detected in REBA assay. Among the 5 infections of SCC samples, HPV 16 (40.0\%), HPV 18 (20.0\%), HPV 53 (20.0\%), HPV 54 (20.0\%) were detected. Among the 64 infections of HSIL samples, HPV 16 (64.1\%), HPV 33 (9.4\%), HPV 58 (7.8\%), HPV 53 and 56 (4.7\% each), HPV 


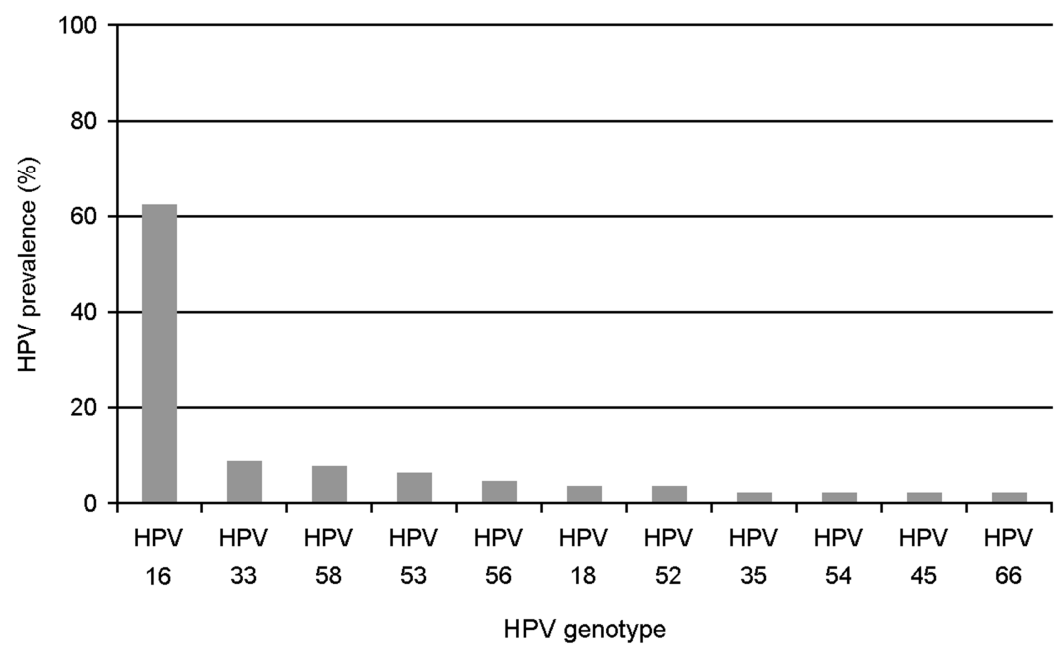

Fig. 1. Prevalence of fifteen HR-HPVs among HSIL and SCC samples $(n=69)$. HPV $16(62.3 \%)$, HPV 33 (8.7\%), HPV 58 (7.3\%), HPV 53 (5.8\%), HPV 56 (4.4\%), HPV 18 and HPV $52(2.9 \%)$, HPV 35, HPV 54, HPV 45 and HPV 66 $(1.5 \%$ each) were detected by REBA.
52 (3.1\%), HPV 18, 35, 45, and 66 (1.6\% each) were detected. Among the 14 infections of Normal samples, HPV18 (35.7\%), HPV 16 (21.4\%), HPV 11 (14.3\%), HPV 35, HPV 52, HPV 66, HPV 54 (7.1\% each) were detected (Table 4).

\section{HPV genotype distribution in high grade cervical lesion}

The distribution of these HPV genotypes varied in high grade cervical lesion, HSIL and SCC. Among the total of 69 HSIL and SCC samples, HPV 16 (62.3\%), HPV 33 (8.7\%), HPV 58 (7.3\%), HPV 53 (5.8\%), HPV 56 (4.4\%), HPV 18 and HPV 52 (2.9\%), HPV 35, HPV 54, HPV 45 and HPV 66 (1.5\% each) were detected in REBA assay (Fig. 1).

\section{DISCUSSION}

HPV DNA testing (The REBA HPV-ID ${ }^{\circledR}$ assay) is used to analyze HPV prevalence and genotype distribution. The overall HPV infection rate in the samples was $100 \%(4 / 4)$, 92.5\% (62/67), 94.7\% (18/19), 93.2\% (55/59), 82.3\% (79/ 86 ) and $51.9 \%$ (41/79) in SCC, HSIL, ASC-H, LSIL, ASCUS, and normal cytology samples, respectively (Table 2). The nearer the normal group, the higher HPV positive rate; however, HPV was detected in more than half the individuals in the normal group (51\%). These data are concordant with our previous report that assessed HPV in the
Korea (Kim et al., 2012b). Additionally, the positivity of HR-HPV infection in the normal group was analyzed and was found to be $13.9 \%$ (Table 2). Other studies have shown similar the positivity of HR-HPV infection in a normal group was $16.8 \%, 27.3 \%$ and $31.7 \%$, respectively (Ding et al., 2014) (Liu et al., 2014) (Sun et al., 2014). The rate of HR-HPV infection increased remarkably in proportion to the severity of the lesion. Among $13.9 \%$ of positive HRHPV infection cases in normal samples, HPV 16 was the most predominant genotype, followed by HPV 11, HPV 35, HPV 52, HPV 54, and HPV 66.

HR- \& LR-HPV mixed infection cases were 13 cases and mostly infected with HR-HPV genotypes such as HPV 16, HPV 18, HPV 33, HPV 35, HPV 53, and HPV 84 (Table $3)$. LR single infection rarely appeared, and its types (HPV 6, HPV 11, HPV 40, HPV 43, and HPV 54) are commonly associated with genital warts. In comparison, the HPVpositive rate of SCC \& HSIL \& ASC-H (93.3\%) was relatively higher than that of ASC-US \& LSIL (86.4\%). Most HPV genotypes detected in SCC \& HSIL were HRHPV genotypes such as HPV 16, HPV 33, HPV 58, HPV 53, HPV 35, and HPV 18 (Table 4). Prevalence of these HR-HPV genotypes was increased in SCC \& HSIL. From LSIL to SCC, however, the detection rate of LR-HPV genotypes decreased. The overall prevalence of HR-HPV infection was $45.7 \%$ (148/324) in all cytological samples, while the overall prevalence of LR-HPV infection and HR- 
\& LR-HPV multiple infection cases were 2.2\% (7/324) and 4.0\% (13/324), respectively (Table 3 ). The single HPV genotype infection rate increased in proportion to severity of lesion, which was found in another study (Okadome et al., 2014).

In order of decreasing HPV genotype prevalence, the most common HPV genotypes in SCC, HSIL and ASC-H were HPV 16, HPV 33, HPV 58, HPV 53, HPV 35, HPV 18, HPV 56, HPV 52, HPV 54, HPV 45, HPV 66, HPV 6, and HPV 11 (Table 4). HPV 16 had a high prevalence of $58.4 \%$ among women with SCC, HSIL and ASC-H in the Western Shandong Province, East China. HPV 33, HPV 58 , HPV 53, and HPV 35 represented $84.3 \%$ of SCC, HSIL and ASC-H. Cervical lesions in close proximity to cancer such as SCC, HSIL and ASC-H can be accounted for by 13 HPV genotypes. This result suggests that the preventive vaccine needs to include more than two HPV genotypes (HPV 16 and 18). Other meta-analysis studies widely support this result (Bao et al., 2008).

HPV 16, HPV 53, HPV 18, HPV 58, HPV 33, and HPV 66 were the most prevalent HPV genotypes in infected women, but the distribution of these HPV genotypes varied with cytological diagnosis (Table 4). Among women with high-grade intraepithelial neoplasia, HPV 16 is still the most prevalent genotype worldwide, but greater geographic variability has been observed for other HPV genotypes. For instance, HPV 31 and HPV 33 get the second and third rank as most prevalent HPVs in Africa and Europe, respectively. On the contrary, HPV 58 and HPV 52 are the second and third most common HPV genotypes found in Asia (Bosch et al., 2008). HPV 53 ranked interestingly in both women with normal cytology and moderate and severe dysplasia group (SCC, HSIL, and ASC-H). HPV 53 is usually detected with normal cytology in each continent (Bosch et al., 2008) and also the third most common genital type of HPV among women in the United States (Hu et al., 2011). However it is not detected with HSIL and SCC in the most countries (Bosch et al., 2008).

In conclusion, the findings of this study suggest that a preventive vaccine against HPV 16 and HPV 18 is not optimal for populations in Eastern China. If a preventive vaccine included HPV 53 and HPV 58, the vaccine efficacy could be increased in this province, based on the results of this study. These data may provide guidance for national or regional vaccination programs in this province to reduce substantially the burden of cervical lesions and HPV infections.

\section{Conflict of interests}

The authors declare that there are no conflicts of interest.

\section{REFERENCES}

Agarossi A, Ferrazzi E, Parazzini F, Perno CF, Ghisoni L. Prevalence and type distribution of high-risk human papillomavirus infection in women undergoing voluntary cervical cancer screening in Italy. J Med Virol. 2009. 81: 529-535.

Bao YP, Li N, Smith JS, Qiao YL. Human papillomavirus type distribution in women from Asia: a meta-analysis. Int $\mathrm{J}$ Gynecol Cancer. 2008. 18: 71-79

Bosch FX, Burchell AN, Schiffman M, Giuliano AR, de Sanjose S, Bruni L, Tortolero-Luna G, Kjaer SK, Muñoz N. Epidemiology and natural history of human papillomavirus infections and type-specific implications in cervical neoplasia. Vaccine. 2008. Suppl 10: K1-16.

Bosch FX, de Sanjose S. Chapter 1: human papillomavirus and cervical cancer - burden and assessment of causality. J Natl Cancer Inst Monogr. 2003. 31: 3-13.

Bouvard V, Baan R, Straif K, Grosse Y, Secretan B, El Ghissassi F, Benbrahim-Tallaa L, Guha N, Freeman C, Galichet L, Cogliano $\mathrm{V}$. A review of human carcinogens-part B: biological agents. Lancet Oncol. 2009. 10: 321-322.

Ding X, Liu Z, Su J, Yan D, Sun W, Zeng Z. Human papillomavirus type-specific prevalence in women referred for colposcopic examination in Beijing. J Med Virol. 2014. 86: 1937 $-1943$.

Dunne EF, Unger ER, Sternberg M, McQuillan G, Swan DC, Patel SS, Markowitz LE. Prevalence of HPV infection among females in the United States. JAMA. 2007. 297: 813-819.

Ferlay J, Shin HR, Bray F, Forman D, Mathers C, Parkin DM Estimates of worldwide burden of cancer in 2008: Globocan 2008. Int J Cancer. 2010. 127: 2893-2917.

Hu SY, Hong Y, Zhao FH, Lewkowitz AK, Chen F, Zhang WH, Pan QJ, Zhang X, Fei C, Li H, Qiao YL. Prevalence of HPV infection and cervical intraepithelial neoplasia and attitudes towards HPV vaccination among Chinese women aged 18 
25 in Jiangsu province. Chin J Cancer Res. 2011. 23: 25-32.

Kim S, Lee D, Park S, Kim TU, Jeon BY, Park KH, Lee H. REBA HPV-ID ${ }^{\circledR}$ for efficient genotyping of human papillomavirus in clinical samples from Korean patients. J Med Virol. 2012a. 84: 1248-1253.

Kim S, Lee D, Kim Y, Kim G, Park S, Choi Y, Kim TU, Park KW, Lee H. Clinical evaluation of human papillomavirus DNA genotyping assay to diagnose women cervical cancer. J Exp Biomed Sci. 2012b. 18: 123-130.

Liu XX, Fan XL, Yu YP, Ji L, Yan J, Sun AH. Human papillomavirus prevalence and type-distribution among women in Zhejiang province, southeast china: across-sectional study. BMC Infectious Diseases. 2014. 14: 708-714.

Okadome M1, Saito T, Tanaka H, Nogawa T, Furuta R, Watanabe
K, Kita T, Yamamoto K, Mikami M, Takizawa K. Potential impact of combined high- and low-risk human papillomavirus infection on the progression of cervical intraepithelial neoplasia 2. J Obstet Gynaecol Res. 2014. 40: 561-569.

Sun B, He J, Chen X, He M, He Z, Wang Y, Shang Q, Yu L, Wei L. Prevalence and genotype distribution of human papillomavirus infection in harbin, Northeast China. Arch Virol. 2014. 159: 1027-1032.

Zhao FH, Lewkowitz AK, Hu SY, Chen F, Li LY, Zhang QM, Wu RF, Li CQ, Wei LH, Xu AD, Zhang WH. Prevalence of human papillomavirus and cervical intraepithelial neoplasia in China: a pooled analysis of 17 population-based studies. Int J Cancer. 2012. 131: 2929-2938. 